\title{
The Range of a Simple Random Walk on $\mathbb{Z}$ : An Elementary Combinatorial Approach
}

\author{
Bernhard A. Moser* \\ Software Competence Center Hagenberg \\ A-4232 Hagenberg, Austria \\ bernhard.moser@scch.at
}

Submitted: Feb 12, 2014; Accepted: Sep 29, 2014; Published: Oct 9, 2014

Mathematics Subject Classifications: 05C81, 60G50

\begin{abstract}
Two different elementary approaches for deriving an explicit formula for the distribution of the range of a simple random walk on $\mathbb{Z}$ of length $n$ are presented. Both of them rely on Hermann Weyl's discrepancy norm, which equals the maximal partial sum of the elements of a sequence. By this the original combinatorial problem on $\mathbb{Z}$ can be turned into a known path-enumeration problem on a bounded lattice. The solution is provided by means of the adjacency matrix $\mathbf{Q}_{d}$ of the walk on a bounded lattice $(0,1, \ldots, d)$. The second approach is algebraic in nature, and starts with the adjacency matrix $\mathbf{Q}_{d}$. The powers of the adjacency matrix are expanded in terms of products of non-commutative left and right shift matrices. The representation of such products by means of the discrepancy norm reveals the solution directly.
\end{abstract}

Keywords: random walk, discrepancy norm, lattice path-enumeration

\section{Introduction}

The problem of determining the distribution of the range of a simple random walk has been treated extensively in the literature. Feller [5] computes the distribution of the range of a standard Brownian motion and derives estimates for the discrete case. In this article he points out that the problem of finding exact formulae for the distribution of the range is difficult to solve in the discrete case. The asymptotic behaviour of the range is investigated, e.g., by [4], [7], [13]. In 1996 the problem was solved by P. Vallois by exploiting martingale techniques [14].

*Supported by Austrian COMET Program. 
In this paper we demonstrate that Feller's problem can be solved in an elementary and concise manner. We present two approaches, both of them rely on Hermann Weyl's discrepancy measure [15]. The first approach, Section 2, exploits the fact that the range of the partial sums of the elements of a sequence defines a norm, the discrepancy norm. The $n$-dimensional unit balls of this norm can be characterized as a zonotope. This allows us to turn the original combinatorial problem on $\mathbb{Z}$ into a known path-enumeration problem on a bounded lattice $L_{d}=(0,1, \ldots, d)$. The solution is expressed in terms of the adjacency matrix $\mathbf{Q}_{d}$ of the corresponding bounded walk. The second approach, Section 4, exploits the algebraic structure of the adjacency matrix $\mathbf{Q}_{d}$ by representing it as sum of a left and a right shift matrix, $\mathbf{Q}_{d}^{-}$and $\mathbf{Q}_{d}^{+}$, respectively. It is shown that a product of these non-commutative matrices can be represented in terms of the discrepancy norm of the sequence of the corresponding signs, -1 and +1 , respectively. This leads to the intuitive Lost Walker Lemma, which immediately provides the solution.

\section{Discrepancy Norm}

Let us consider a path $\gamma=\left((0,0)^{T},\left(1, x_{1}\right)^{T},\left(1, x_{1}\right)^{T}+\left(1, x_{2}\right)^{T}, \ldots, \sum_{i=1}^{n}\left(1, x_{i}\right)^{T}\right)$ in $\left(\mathbb{N}_{0} \times\right.$ $\mathbb{R})^{n+1}$. The diameter (range) of $\gamma$ in the direction of $(0,1)^{T}$ is given by

$$
\max _{1 \leqslant n_{1} \leqslant n_{2} \leqslant n}\left|\sum_{i=n_{1}}^{n_{2}}\left\langle\left(1, x_{i}\right)^{T},(0,1)^{T}\right\rangle\right|=\max _{1 \leqslant n_{1}, n_{2} \leqslant n}\left|\sum_{i=n_{1}}^{n_{2}} x_{i}\right|=\max _{1 \leqslant i \leqslant n} \sum_{j=0}^{i} x_{j}-\min _{1 \leqslant i \leqslant n} \sum_{j=0}^{i} x_{j},
$$

where $\langle.,$.$\rangle denotes the usual inner product, and x_{0}=0$. Hermann Weyl [15] introduced the measure

$$
\|\cdot\|_{D}: \mathbb{R}^{n} \rightarrow \mathbb{R}_{0}^{+},\left(x_{1}, \ldots, x_{n}\right) \mapsto \max _{1 \leqslant a \leqslant b \leqslant n}\left|\sum_{i=a}^{b} x_{i}\right|,
$$

in the context of measuring irregularities of distributions. It satisfies the axioms of a norm and was investigated in the context of numerical integration [12], computational geometry [2], pattern recognition [11], image processing [1, 8] and level crossing sampling [10].

First of all let us prove some fundamental properties of the discrepancy norm. We call $J$ a minimal interval with maximal discrepancy (MMD), if $\left|\sum_{k \in J} x_{k}\right|=\|\mathbf{x}\|_{D}=d$ and if $L \subsetneq J$ implies $\left|\sum_{k \in L} x_{k}\right|<d$, where $L$ is a subinterval of $J$. Note that MMD intervals are mutually disjoint. Let $K$ denote the number of MMD intervals, and let us enumerate these $K$ MMD intervals, $\left(J_{j}\right)_{j=1, \ldots, K}$, in an increasing order, such that $j_{1}<j_{2}$ implies $a<b$ for all $a \in J_{j_{1}}$ and $b \in J_{j_{2}}$. By this we obtain the partition of subintervals

$$
\{1, \ldots, n\}=\widetilde{J}_{0} \cup \bigcup_{i=1}^{K}\left(J_{j} \cup \widetilde{J}_{j}\right)
$$

with MMD intervals $J_{j}=\left\{a_{j}, \ldots, b_{j}\right\}$, and $\left|\sum_{k \in \widetilde{J}_{l}} x_{k}\right|<d, l=0, \ldots, K$. Observe that, in analogy to the Chebychev alternation theorem, the signs $\left(\sigma_{j}\right)_{j=1, \ldots, K} \in\{-1,1\}^{K}$ of the sums $\sum_{k \in J_{j}} x_{k}$ are alternating. As a direct consequence of the MMD property we obtain Lemma 1. 


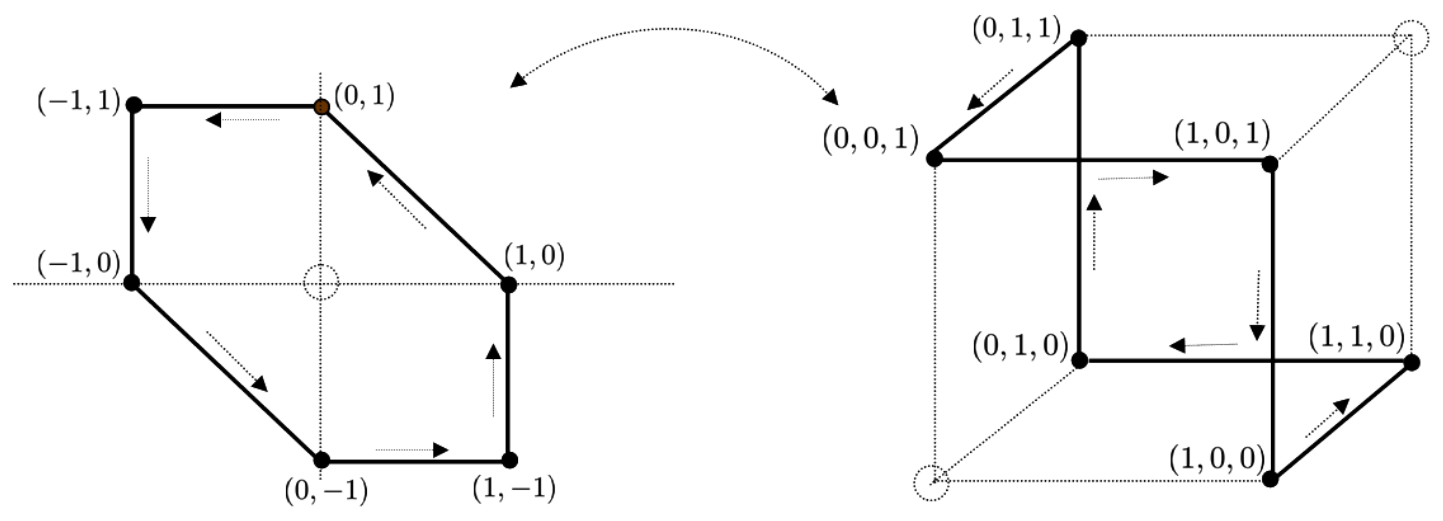

Figure 1: Illustration of bijection between the set of vertices of the $n$-dimensional unit ball of the discrepancy norm and those of the $(n+1)$-dimensional hypercube without the diaognal elements for $n=2$.

Lemma 1 (MMD Lemma). Let $n, d \in \mathbb{N}, \mathbf{x}=\left(x_{i}\right)_{i=1, \ldots, n} \in\{-1,1\}^{n}$. Let $\left(J_{k}\right)_{k=1, \ldots, K}$, $J_{k}=\left\{a_{k}, \ldots, b_{k}\right\}$, be the MMD intervals in increasing order, and $\left(\sigma_{k}\right)_{k=1, \ldots, K}$ the corresponding signum values of the partial sums over the MMD intervals. Then, for any $k \in\{1, \ldots, K\}$ there holds $\sigma_{k} \sum_{j=a_{k}}^{i} x_{k}>0$ and $\sigma_{k} \sum_{j=0}^{b_{k}-i} x_{b_{k}-j}>0$ for all $i \in\left\{a_{k}, \ldots, b_{k}\right\}$.

The geometric approach of Section 3 is motivated by the characterization of the $n$ dimensional unit ball of $\|\cdot\|_{D}$ by means of a zonotope, that is a projection mapping from the hypercube $[0,1]^{n+1}$, see $[9]$. The unit ball of $\|\cdot\|_{D}$ turns out to be a polytope, whose vertices 1-1 correspond to the vertices of the hypercube $[0,1]^{n+1}$ without the elements $\mathbf{0}=(0,0, \ldots, 0)$ and $\mathbf{1}=(1,1, \ldots, 1)$. See Figure 1 for an illustration for $n=2$. Lemma 2 formulates this result in terms of walks comprising -1 and 1 steps with range $d$. Though Lemma 2 can be directly followed from the zonotope characterization theorem of [9], we provide an alternative self-contained proof.

Lemma 2 (Zonotope Lemma). Let $n, d \in \mathbb{N}$ and $\mathbf{x}=\left(x_{1}, \ldots, x_{n}\right) \in\{-1,1\}^{n}$. Then,

$$
\begin{aligned}
\|\mathbf{x}\|_{D}=d \Longleftrightarrow & \exists_{1} z_{1}, \ldots, z_{n+1} \in\{0,1, \ldots, d\}: x_{i}=z_{i+1}-z_{i}, \\
& \left|z_{i+1}-z_{i}\right|=1, \min _{i} z_{i}=0, \max _{i} z_{i}=d .
\end{aligned}
$$

Proof. First of all, note that, if there is a representation of $\mathbf{x}$ such that $\|\mathbf{x}\|_{D}=d$, then, in terms of $z_{n+1} \in\{0,1, \ldots, d\}: x_{i}=z_{i+1}-z_{i},\left|z_{i+1}-z_{i}\right|=1, \min _{i}\left\{z_{i}\right\}=0, \max _{i}\left\{z_{i}\right\}=d$, it is unique. This follows from the fact that the equations $x_{i}=z_{i+1}-z_{i}$ are determined up to one degree of freedom. With the additional property $\min _{i} z_{i}=0$, we see that no degree of freedom remains.

The $\Leftarrow$ implication is immediately checked by considering $z_{h}=\min _{i} z_{i}=0, z_{k}=$ $\max _{i} z_{i}=d$ and $\left|\sum_{i=\min \{h, k\}}^{\max \{h, k\}-1} z_{i+1}-z_{i}\right|=\left|z_{h}-z_{k}\right|=d$.

In order to prove the $\Rightarrow$ implication let us assume $\|\mathbf{x}\|_{D}=d$ and the notation of the MMD Lemma 1. 
Next we construct a sequence $\left(z_{1}, \ldots, z_{n+1}\right) \in\{0, \ldots, d\}^{n+1}$ such that $x_{i}=z_{i+1}-z_{i}$, $\left|z_{i+1}-z_{i}\right|=1, \min _{i} z_{i}=0$, and $\max _{i} z_{i}=d$.

We distinguish two cases $\sigma_{1}= \pm 1$ :

Case 1 In case of $\sigma_{1}>0$ let us set $z_{a_{1}}=0, z_{i}=\sum_{j=a_{1}}^{i-1} x_{j}$ if $i \in\left\{a_{1}, \ldots, n+1\right\}$, and $z_{a_{1}-i}=-\sum_{j=1}^{i} x_{a_{1}-j}$ if $a_{1}>1$ and $i \in\left\{1, \ldots, a_{1}-1\right\}$. Elementary algebra shows that $x_{i}=z_{i+1}-z_{i}$. The assumption $\|\mathbf{x}\|_{D} \leqslant d$ immediately implies $\forall i: z_{i} \leqslant d$.

Now, suppose that $\sigma_{1}>0$ and $z_{i}<0$. If $i \in \widetilde{J}_{0}$ then $z_{i}=-\sum_{j=i}^{a_{1}-1} x_{j}<0$, hence $0<\sum_{j=i}^{a_{1}-1} x_{j}$ and $\sum_{j=i}^{b_{1}} x_{j}>d$, which contradicts the assumed MMD property of $J_{1}$. If $i \in \widetilde{J}_{k}, k>0$, then $z_{i}=\sum_{1}+\sum_{2}$, where $\sum_{1}=\sum_{j=a_{1}}^{b_{k}} x_{j}$ and $\sum_{2}=\sum_{j=b_{1}+1}^{i} x_{j}$.

For even $k$ we have $\sum_{1}=0$, hence, $\sum_{j=a_{k}}^{b_{k}} x_{j}=-d$ and $\sum_{2}<0$. But then we get $\sum_{j=a_{k}}^{i} x_{j}<-d$, which contradicts the MMD property of $J_{k}$.

For odd $k$ we have $\sum_{1}=d$. The assumption $z_{i}<0$ implies $\sum_{1}+\sum_{2}<0$, hence, $\sum_{2}<-d$, which contradicts the assumption that $\|\mathbf{x}\|_{D} \leqslant d$.

Now, consider $i \in J_{k}$. First, assume that $k$ is even. Then, $z_{i}+\sum_{j=i+1}^{b_{k}} x_{j}=0$, hence $\sum_{k=i+1}^{b_{k}} x_{j}>0$. On the other hand, we have $-d=\sum_{j=a_{k}}^{b_{k}} x_{j}=\sum_{j=a_{k}}^{i} x_{j}+\sum_{j=i+1}^{b_{k}} x_{j}$, hence $\sum_{j=a_{k}}^{i} x_{j}<-d$, which contradicts $\|\mathbf{x}\|_{D} \leqslant d$. Next, assume that $k$ is odd. Then, $\sum_{j=a_{1}}^{b_{k}} x_{j}=d=z_{i}+\sum_{j=i+1}^{b_{k}} x_{j}$ entails $\sum_{j=i+1}^{b_{k}} x_{j}>d$, which contradicts $\|\mathbf{x}\|_{D} \leqslant d$. From this we conclude that $z_{i} \geqslant 0$.

Case 2 In case of $\sigma_{1}<0$ let us set $z_{a_{1}}=d, z_{i}=\sum_{j=a_{1}}^{i-1}\left(d+x_{j}\right)$ if $i \in\left\{a_{1}, \ldots, n+1\right\}$, and $z_{a_{1}-i}=d-\sum_{j=1}^{i} x_{a_{1}-j}$ if $a_{1}>1$ and $i \in\left\{1, \ldots, a_{1}-1\right\}$. Again, elementary algebra yields $x_{i}=z_{i+1}-z_{i}$, and, analogous reasoning as in case 1 shows that $z_{i} \geqslant 0$.

The algebraic approach of Section 4 relies on the following Lemma 3, by which the discrepancy norm of a binary walk can be determined by means of a tree. See Figure 3 for an illustration.

Lemma 3 (Zero-Bounded Subtraction Lemma). Let $\tau: \bigcup_{n \geqslant 0}\{-1,1\}^{n} \rightarrow \mathbf{N}_{0}$ be defined by $\tau(\emptyset)=0, \tau(\mathbf{x},+1)=\tau(\mathbf{x})+1$ and $\tau(\mathbf{x},-1)=(\tau(\mathbf{x})-1)^{+}$, where $(t)^{+}=$ $\max \{t, 0\}$. Then

$$
\|\mathbf{x}\|_{D}=\tau(\mathbf{x})+\tau(-\mathbf{x}) .
$$

Proof. Given $\mathbf{x} \in\{-1,1\}^{n}, n \in \mathbf{N}, d=\|\mathbf{x}\|_{D}$ and $1 \leqslant i \leqslant n$, we define $\tau(\mathbf{x}, i)=$ $\tau\left(\left(x_{1}, \ldots, x_{i}\right)\right)$. Note that for $1 \leqslant i_{1} \leqslant i_{2} \leqslant n$ there holds

$$
\sum_{j=i_{1}}^{i_{2}} x_{j} \leqslant \tau\left(\mathbf{x}, i_{2}\right) \leqslant\|\mathbf{x}\|_{D} .
$$




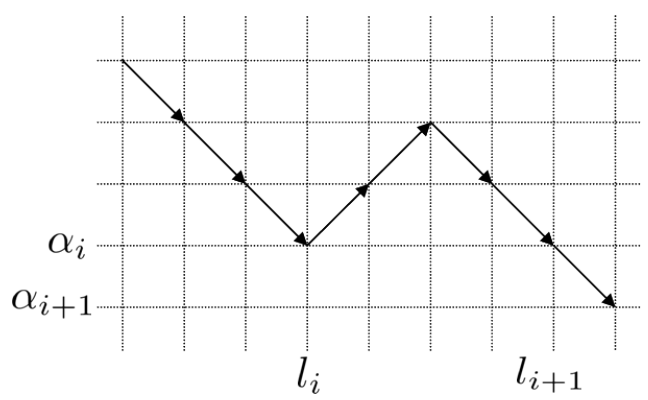

Figure 2: Illustration of definition (8) for $\sigma_{k}<0$, i.e., for a decreasing sequence of partial sums $\alpha_{i}=\sum_{j=a_{k}}^{l_{i}} x_{j}$.

Using the notation of Lemma 3, equation (6) implies

$$
\sum_{j=a_{k}}^{b_{k}} x_{j}=d=\tau\left(\mathbf{x}, b_{k}\right)
$$

for $\sigma_{k}>0$. Next, we show that $\tau\left(\mathbf{x}, b_{k}\right)=0$ if $\sigma_{k}<0$. For $d=1$ the sequence $\mathbf{x}$ is alternating in sign. Therefore, we have $k=a_{k}=b_{k}$, which immediately implies $\tau(\mathbf{x}, k)=\left(\sigma_{k}+1\right) / 2$. For $d>1$ consider $l_{1}=a_{k}$ and

$$
l_{i}=\min \left\{l \geqslant l_{i-1}|| \sum_{j=a_{k}}^{l} x_{j}|=| \sum_{j=a_{k}}^{l_{i-1}} x_{j} \mid+1\right\}
$$

for $2 \leqslant i \leqslant d$. Then, observe that

$$
\sum_{j=l_{i}+1}^{k} x_{j} \geqslant 0
$$

for any $k \in\left\{l_{i}+1, \ldots, l_{i+1}-1\right\}$. See Figure 2 for an illustration. (9) implies

$$
\tau\left(\mathbf{x}, l_{i}\right)=\tau\left(\mathbf{x}, l_{i+1}-1\right)
$$

for $1 \leqslant i \leqslant d-1$. Due to $\sigma_{k}<0$ and the fact, that $\left\{a_{k}, \ldots, b_{k}\right\}$ is an MMD interval, the corresponding sequence $\alpha_{i}=\sum_{j=a_{k}}^{l_{i}} x_{j}$ is strictly decreasing from $\alpha_{1}=-1$ to $\alpha_{1}=-d$. Because of (6) there is an index $i_{0} \in\{1, \ldots, d\}$, such that

$$
\tau\left(\mathbf{x}, a_{k}-1\right)+\sum_{j=l_{1}}^{l_{i_{0}}} x_{j}=\tau\left(\mathbf{x}, l_{i_{0}}\right)=0 .
$$

Now, let us apply induction. Suppose that $\tau\left(\mathbf{x}, l_{i}\right)=0$. Taking (10) into account yields $\tau\left(\mathbf{x}, l_{i}\right)=\tau\left(\mathbf{x}, l_{i+1}-1\right)=0$. Since the sequence $\left(\alpha_{i}\right)_{i}$ is strictly decreasing, we obtain $\tau\left(\mathbf{x}, l_{i+1}\right)=0$. From this it follows that $\tau\left(\mathbf{x}, l_{d}\right)=\tau\left(\mathbf{x}, b_{k}\right)=0$.

As a result we obtain $\tau\left(\mathbf{x}, b_{k}\right)=d$, if $\sigma_{k}>0$, and $\tau\left(\mathbf{x}, b_{k}\right)=0$, if $\sigma_{k}<0$. Note that $\mathbf{x}$ and $-\mathbf{x}$ have the same MMD intervals but with flipping signs, i.e., $\sigma_{k}(\mathbf{x})=-\sigma_{k}(-\mathbf{x})$, 


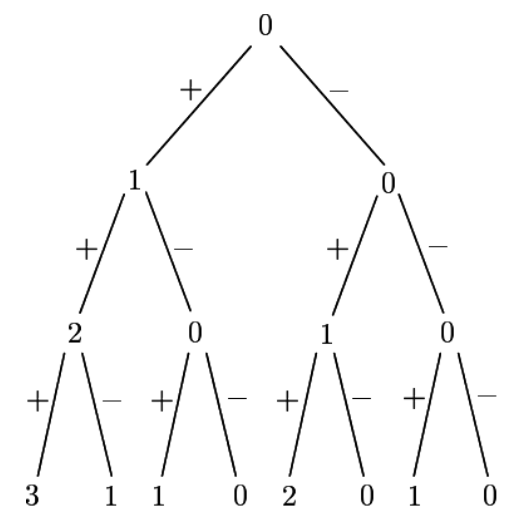

Figure 3: Illustration of the tree $\tau$ in Lemma 3. Flipping the signs along a branch $\mathbf{x} \in\{-1,1\}^{n}$ in the tree and adding the corresponding leaves yields the discrepancy norm $\|\mathbf{x}\|_{D}=\tau(-\mathbf{x})+\tau(\mathbf{x})$. E.g., $\|(++-)\|_{D}=\tau(++-)+\tau(--+)$.

where $\sigma_{k}(\mathbf{x})$ denotes the signum of the $k$-th MMD interval induced by $\mathbf{x}$. From this it follows that

$$
\tau\left(\mathbf{x}, b_{K}\right)+\tau\left(-\mathbf{x}, b_{K}\right)=d .
$$

If $b_{K}=n$ we have finished. Otherwise, suppose that $b_{K}<n$. Without loss of generality we may assume that $\sigma_{K}>0$. The MMD property of the interval $\left\{a_{K}, \ldots, b_{k}\right\}$ and the fact that $K$ is the last MMD interval, implies that $-d<\sum_{j=b_{K}+1}^{i} x_{j} \leqslant 0$ for all $i \in\left\{b_{K}+1, \ldots, n\right\}$, hence

$$
\tau(\mathbf{x}, n)=d+\sum_{j=b_{K}+1}^{n} x_{j} .
$$

Next, consider $-\mathbf{x}$ such that $\sigma_{K}(-\mathbf{x})=-\sigma_{K}(\mathbf{x})=-1$. The MMD property guarantees that $\sum_{j=b_{K}+1}^{i}\left(-x_{j}\right) \geqslant 0$ for $i \in\left\{b_{K}+1, \ldots, n\right\}$, hence

$$
\tau(-\mathbf{x}, n)=0+\sum_{j=b_{K}+1}^{n}\left(-x_{j}\right) .
$$

Combining results (13) and (14) demonstrates the truth of (5).

\section{Geometric Approach}

Lemma 2 relates the problem of determining $\#\left\{\mathbf{x} \in\{-1,1\}^{n} \mid\|\mathbf{x}\|_{D} \leqslant d\right\}$ with the pathenumeration problem $\#\left\{\left(z_{1}, \ldots, z_{n+1}\right) \in\{0, \ldots, d\}^{n+1}|| z_{i+1}-z_{i} \mid=1\right\}$ on the bounded lattice $L_{d}=(0, \ldots, d)$. The enumeration of paths of walks comprising -1 and 1 steps on a bounded lattice $L_{d}$ is well understood by means of the corresponding $(d+1) \times(d+1)$ 
adjacency matrix $\mathbf{Q}_{d+1}$, see, e.g., [3, 6],

$$
\mathbf{Q}_{d+1}=\left(\begin{array}{cccccc}
0 & 1 & 0 & \cdots & \cdots & 0 \\
1 & 0 & 1 & \ddots & \ddots & 0 \\
0 & 1 & 0 & \ddots & \ddots & \vdots \\
\vdots & \ddots & \ddots & \ddots & \ddots & 0 \\
\vdots & \ddots & \ddots & \ddots & \ddots & 1 \\
0 & 0 & \cdots & 0 & 1 & 0
\end{array}\right)
$$

From [6] the total number of different paths on $L_{d}$ of length $n$ starting at level $j \in L_{d}$ and ending at level $k \in L_{d}$ is given by $\mathbf{e}_{j}^{T} \mathbf{Q}_{d+1}^{n} \mathbf{e}_{k}$. This implies Lemma 4 , where $\mathbf{1}=(1, \ldots, 1)^{T}$ :

Lemma 4. Let $n, d \in \mathbb{N}$, then $\#\left\{\left(z_{1}, \ldots, z_{n+1}\right) \in\{0, \ldots, d\}^{n+1}|| z_{i+1}-z_{i} \mid=1\right\}=$ $\mathbf{1}^{T} \mathbf{Q}_{d+1}^{n} \mathbf{1}$.

Taking Lemma 2 and Lemma 4 together immediately yields the solution for the enumeration of symmetric walks with maximal range $d$, as presented in Theorem 5 .

Theorem 5. Let $n, d \in \mathbb{N}$, then $\#\left\{\mathbf{x} \in\{-1,1\}^{n} \mid\|\mathbf{x}\|_{D} \leqslant d\right\}=\mathbf{1}^{T} \mathbf{Q}_{d+1}^{n} \mathbf{1}-\mathbf{1}^{T} \mathbf{Q}_{d}^{n} \mathbf{1}$.

Proof. Note, that

$$
\begin{aligned}
\mathbf{1}^{T} \mathbf{Q}_{d+1}^{n} \mathbf{1}-\mathbf{1}^{T} \mathbf{Q}_{d}^{n} \mathbf{1} & =\#\left\{\left(z_{i}\right)_{i} \in\{0, \ldots, d\}^{n+1}|| z_{i+1}-z_{i} \mid=1, \max _{i} z_{i}=d\right\} \\
& =\# \bigcup_{k=0}^{d}\left\{\left(z_{i}\right)_{i} \in\{0, \ldots, d\}^{n+1}|| z_{i+1}-z_{i} \mid=1, \max _{i} z_{i}=d, \min _{i} z_{i}=k\right\} \\
& =\#\left\{\mathbf{x} \in\{-1,1\}^{n} \mid\|\mathbf{x}\|_{D} \leqslant d\right\}
\end{aligned}
$$

where (16) follows from Lemma 4 and (17) is a consequence of Lemma 2.

\section{Algebraic Approach}

For ease of notation, let $\mathbf{0}_{k}=(0, \ldots, 0)$ denote the zero vector with $k$ entries, and, analogously, $\mathbf{1}_{k}=(1, \ldots, 1)$.

Lemma 6 (Lost Walker Lemma). Let $d \in \mathbb{N}$. Let $\mathbf{Q}_{d}^{(-1)}=\left(q_{i, j}\right)_{i, j}$ be the $d \times d$ left shift matrix with 1 in the subdiagonal, i.e., $q_{i-1, i}=1$ and 0 else. Further, let $\mathbf{Q}_{d}^{(+1)}$ be the transpose of $\mathbf{Q}_{d}^{(-1)}$, that is the corresponding right shift matrix. Let $\mathbf{x}=\left(x_{1}, \ldots, x_{n}\right) \in$ $\{-1,1\}^{n}$, then

$$
\left(\mathbf{0}_{\tau(-\mathbf{x})}, \mathbf{1}_{d-(\tau(\mathbf{x})+\tau(-\mathbf{x}))}, \mathbf{0}_{\tau(\mathbf{x})}\right)^{T}=\prod_{i=1}^{n} \mathbf{Q}_{d}^{\left(x_{j}\right)} \mathbf{1} .
$$




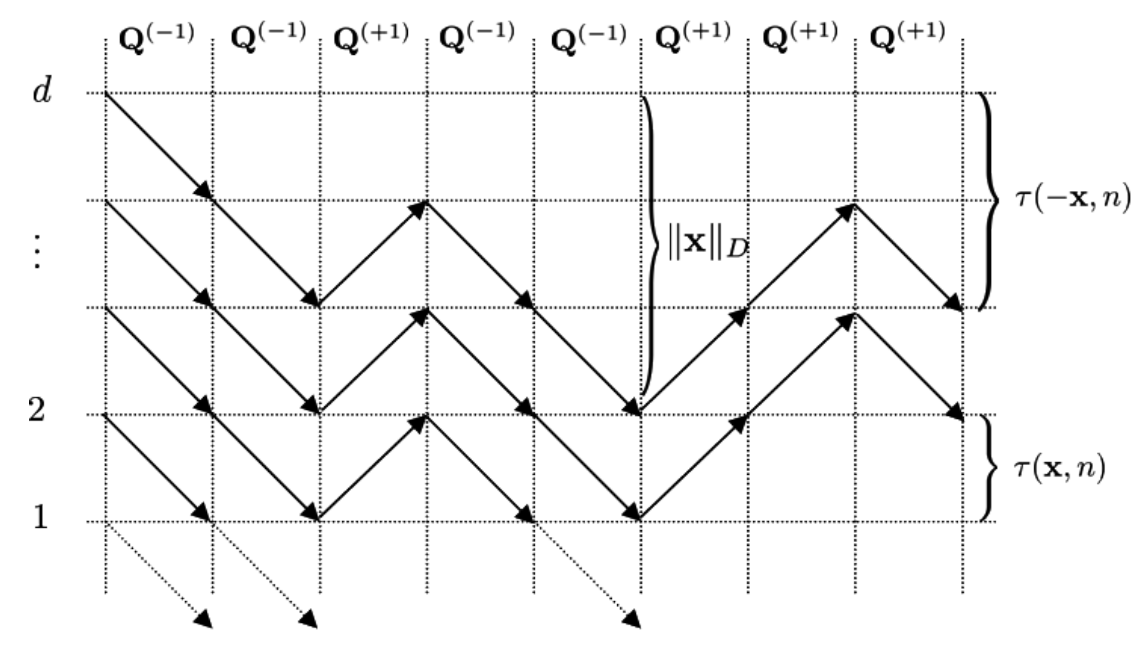

Figure 4: Illustration of equation (18) of the Lost Walker Lemma. The number $\#\{1,2,3\}$ of lost walkers equals the discrepancy norm $\|\mathbf{x}\|_{D}=\tau(-\mathbf{x}, n)+\tau(\mathbf{x}, n)$ of the route $\mathbf{x} \in\{-1,1\}^{n}$.

Proof. Result (18) follows immediately from the $\tau$ recursion defined in Lemma 3 by checking

$$
\begin{aligned}
& \left(\mathbf{0}_{k_{1}+1}, \mathbf{1}_{\left.d-\left(k_{1}+1+\left(k_{2}-1\right)^{+}\right)\right)}, \mathbf{0}_{\left(k_{2}-1\right)^{+}}\right)^{T}=\mathbf{Q}_{d}^{(-1)}\left(\mathbf{0}_{k_{1}}, \mathbf{1}_{\left.d-\left(k_{1}+k_{2}\right)\right)}, \mathbf{0}_{k_{2}}\right)^{T} \\
& \left(\mathbf{0}_{\left(k_{1}-1\right)^{+}}, \mathbf{1}_{\left.d-\left(\left(k_{1}-1\right)^{+}+k_{2}+1\right)\right)}, \mathbf{0}_{k_{2}+1}\right)^{T}=\mathbf{Q}_{d}^{(+1)}\left(\mathbf{0}_{k_{1}}, \mathbf{1}_{\left.d-\left(k_{1}+k_{2}\right)\right)}, \mathbf{0}_{k_{2}}\right)^{T} .
\end{aligned}
$$

(18) can be illustrated by considering a group of walkers, where each unit vector $\mathbf{e}_{i}$ represents a walker starting at level $i$. $\mathbf{x}$ controls the route for the walk in the bounded lattice $(1, \ldots, d)$. See Figure 4 for an illustration. If the route for a walker leads outside the lattice, this walker gets lost. The discrepancy norm $\|\mathbf{x}\|_{D}$ equals the total number of lost walkers.

Lemma 6 allows us to represent the expression $\mathbf{1}^{T} \prod_{i=1}^{n} \mathbf{Q}_{d}^{\left(x_{j}\right)} \mathbf{1}$ in terms of the discrepancy norm of $\mathbf{x}$. Corollary 7 is a direct consequence of Lemma 6 .

Corollary 7. Let $d, n \in \mathbb{N}, \mathbf{x}=\left(x_{i}\right)_{i} \in\{-1,1\}^{n}$. Then,

$$
\mathbf{1}^{T} \prod_{i=1}^{n} \mathbf{Q}_{d}^{\left(x_{j}\right)} \mathbf{1}=\left(d-\|\mathbf{x}\|_{D}\right)^{+} .
$$

Theorem 5 follows from Corollary 7 by considering

$$
\begin{aligned}
\mathbf{1}^{T} \mathbf{Q}_{d+1}^{n} \mathbf{1} & =\mathbf{1}^{T}\left(\mathbf{Q}_{d+1}^{(-1)}+\mathbf{Q}_{d+1}^{(+1)}\right)^{n} \mathbf{1} \\
& =\sum_{\left(x_{i}\right)_{i} \in\{-1,1\}^{n}} \mathbf{1}^{T} \prod_{i=1}^{n} \mathbf{Q}_{d+1}^{\left(x_{j}\right)} \mathbf{1} \\
& =\sum_{\mathbf{x} \in\{-1,1\}^{n}}\left(d+1-\|\mathbf{x}\|_{D}\right)^{+} .
\end{aligned}
$$


Equation (20) yields

$$
\begin{aligned}
\mathbf{1}^{T} \mathbf{Q}_{d+1}^{n} \mathbf{1}-\mathbf{1}^{T} \mathbf{Q}_{d}^{n} \mathbf{1} & =\sum_{\mathbf{x} \in\{-1,1\}^{n},\|\mathbf{x}\|_{D} \leqslant d} d+1-\|\mathbf{x}\|_{D}-\sum_{\mathbf{x} \in\{-1,1\}^{n},\|\mathbf{x}\|_{D} \leqslant d-1} d-\|\mathbf{x}\|_{D} \\
& =\sum_{\mathbf{x} \in\{-1,1\}^{n},\|\mathbf{x}\|_{D}=d} 1+\sum_{\mathbf{x} \in\{-1,1\}^{n},\|\mathbf{x}\|_{D} \leqslant d-1} 1 \\
& =\#\left\{\mathbf{x} \in\{-1,1\}^{n} \mid\|\mathbf{x}\|_{D} \leqslant d\right\} .
\end{aligned}
$$

\section{$5 \quad$ Asymmetric Walks}

Consider independent and identically distributed random variables $X_{i}$, each taking a step -1 or +1 with step probabilities $P(X=+1)=p$ and $P(X=-1)=1-p=q$, respectively. $S_{n}=\sum_{j=i}^{n} X_{i}$ defines a random walk on $\mathbf{Z}$ induced by $X_{i}$. Let $\mathbf{X}_{n}=$ $\left(X_{1}, \ldots, X_{n}\right)$, further, let $N_{+}(\mathbf{x})$ denote the number of +1 steps of the walk $\mathbf{x} \in\{-1,1\}^{n}$. Now, we use Theorem 5 to determine the distribution $P\left(\|X\|_{D} \leqslant d\right)$. For this let us introduce the matrix $\mathbf{Q}_{d+1, p}$

$$
\mathbf{Q}_{d+1, p}=\left(\begin{array}{cccccc}
0 & p & 0 & \cdots & \cdots & 0 \\
q & 0 & p & \ddots & \ddots & 0 \\
0 & q & 0 & \ddots & \ddots & \vdots \\
\vdots & \ddots & \ddots & \ddots & \ddots & 0 \\
\vdots & \ddots & \ddots & \ddots & \ddots & p \\
0 & 0 & \cdots & 0 & q & 0
\end{array}\right)
$$

and, analogously, the corresponding left and right shift matrices $\mathbf{Q}_{d+1, p}^{(+1)}$ and $\mathbf{Q}_{d+1, p}^{(-1)}$.

Theorem 8. Let $p \in(0,1)$ and $\mathbf{X}_{n}=\left(X_{1}, \ldots, X_{n}\right)$, where $X_{i}$ are independent and identically distributed random variables with $P\left(X_{i}=+1\right)=p$ and $P\left(X_{i}=-1\right)=1-p$. Then,

$$
P\left(\left\|\mathbf{X}_{n}\right\|_{D} \leqslant d\right)=\mathbf{1}^{T} \mathbf{Q}_{d+1, p}^{n} \mathbf{1}-\mathbf{1}^{T} \mathbf{Q}_{d, p}^{n} \mathbf{1} .
$$

Proof. Corollary 7 yields

$$
\begin{aligned}
\mathbf{1}^{T} \mathbf{Q}_{d+1, p}^{n} \mathbf{1} & =\sum_{k=0}^{n} \sum_{\substack{\mathbf{x} \in\{-1,1\}^{n}, N_{+}(\mathbf{x})=k}} p^{k}(1-p)^{n-k} \mathbf{1}^{T} \prod_{i=1}^{n} Q_{d+1, p}^{\left(x_{i}\right)} \mathbf{1} \\
& =\sum_{k=0}^{n} p^{k}(1-p)^{n-k} \sum_{\substack{\mathbf{x} \in\{-1,1\}^{n} \\
N_{+}(\mathbf{x})=k}}\left(d-\|\mathbf{x}\|_{D}\right)^{+}
\end{aligned}
$$


Result (24) implies, for $d>1$,

$$
\begin{aligned}
\mathbf{1}^{T} \mathbf{Q}_{d+1, p}^{n} \mathbf{1}-\mathbf{1}^{T} \mathbf{Q}_{d, p}^{n} \mathbf{1} & =\sum_{k=0}^{n} p^{k}(1-p)^{n-k}\left(\sum_{\substack{\|\mathbf{x}\|_{D}<d, N_{+}(\mathbf{x})=k}} d-\|\mathbf{x}\|_{D}-\sum_{\substack{\|\mathbf{x}\|_{D}<d-1, N_{+}(\mathbf{x})=k}} d-1-\|\mathbf{x}\|_{D}\right) \\
& \left.=\sum_{k=0}^{n} p^{k}(1-p)^{n-k} \#\left\{\mathbf{x} \in\{-1,1\}^{n} \mid\|\mathbf{x}\|_{D} \leqslant d, N_{+}(\mathbf{x})=k\right)\right\} \\
& =P\left(\left\|\mathbf{X}_{n}\right\|_{D} \leqslant d\right) .
\end{aligned}
$$

\section{Factorization of $\mathrm{Q}_{d, p}$}

From [16] we know that the matrix $\mathbf{Q}_{d, p}$ of Equation (22) may be factorized by way of $\mathbf{Q}_{d, p}=\mathbf{V} \boldsymbol{\Lambda} \mathbf{V}^{-1}$, where $\boldsymbol{\Lambda}=\operatorname{diag}\left(\lambda_{1}, \ldots, \lambda_{d}\right)$ is a diagonal matrix with diagonal elements $\lambda_{i}$ and $\mathbf{V}=\left(\mathbf{v}_{1}, \ldots, \mathbf{v}_{d}\right)$ is a matrix of corresponding eigenvectors given by

$$
\begin{aligned}
\lambda_{k} & =2 \sqrt{p(1-p)} \cos \left(\frac{k \pi}{d+1}\right) \\
\mathbf{v}_{k} & =\frac{\sqrt{2}}{\sqrt{d+1}}\left(\rho^{0} \sin \left(1 \cdot \frac{k \pi}{d+1}\right), \ldots, \rho^{d-1} \sin \left(d \cdot \frac{k \pi}{d+1}\right)\right)^{T},
\end{aligned}
$$

where $\rho=\sqrt{q / p} \geqslant 1,0<p \leqslant 1 / 2$, and $q=1-p$. Because of $\mathbf{V} \mathbf{V}^{T}=\operatorname{diag}\left(\rho^{0}, \rho^{2}, \ldots, \rho^{2 d}\right)$ we get $\mathbf{V}^{-1}=\mathbf{V}^{T} \operatorname{diag}\left(\rho^{0}, \rho^{-2}, \ldots, \rho^{-2 d}\right)$. Consequently, we obtain

$$
\begin{aligned}
\left(\mathbf{V}^{-1} \mathbf{1}\right)^{T} & =\frac{\sqrt{2}}{\sqrt{d+1}}\left(\sum_{k=1}^{d}\left(\frac{1}{\rho}\right)^{k-1} \sin \left(\frac{j k \pi}{d+1}\right)\right)_{j} \\
\mathbf{1}^{T} \mathbf{V} & =\frac{\sqrt{2}}{\sqrt{d+1}}\left(\sum_{k=1}^{d} \rho^{k-1} \sin \left(\frac{j k \pi}{d+1}\right)\right)_{j} .
\end{aligned}
$$

Note that

$$
\sum_{j=1}^{d} a^{j} \sin \left(\frac{k j \pi}{d+1}\right)=a \frac{\left(1-(-1)^{k} a^{d+1}\right) \sin \left(\frac{k \pi}{d+1}\right)}{1-2 a \cos \left(\frac{k \pi}{d+1}\right)+a^{2}} .
$$

Now, (25), (26) and (27), applied on Theorem 5, lead on the closed-form representation of Proposition 9.

Proposition 9. Let $p \in(0,1), q=1-p, n, d \in \mathbb{N}$, and $\mathbf{X}_{n}$ as in Theorem 5 , then

$$
\begin{aligned}
P\left(\left\|\mathbf{X}_{n}\right\|_{D} \leqslant d\right)=\frac{(4 p q)^{\frac{n}{2}+1}}{2} & \left\{\frac{1}{d+2} \sum_{k=1}^{d+1} \kappa_{d+1, k, p} \cos ^{n}\left(\theta_{d+2, k}\right) \chi_{p}\left(\theta_{d+2, k}\right)\right. \\
& \left.-\frac{1}{d+1} \sum_{k=1}^{d} \kappa_{d, k, p} \cos ^{n}\left(\theta_{d+1, k}\right) \chi_{p}\left(\theta_{d+1, k}\right)\right\},
\end{aligned}
$$


where $\theta_{d, k}=\frac{k \pi}{d}, \kappa_{d, k, p}=2-(-1)^{k} \gamma_{d, p}$,

$$
\chi_{p}(\theta)=\frac{1-\cos ^{2}(\theta)}{(1-2 \sqrt{p(1-p)} \cos (\theta))^{2}}, \text { and } \gamma_{d, p}=\left(\frac{p}{q}\right)^{\frac{d+1}{2}}+\left(\frac{q}{p}\right)^{\frac{d+1}{2}} \text {. }
$$

In the special case $p=q=1 / 2$ we obtain $\kappa_{d+1, k, p}=4$ for $k$ odd and 0 else, hence

$$
\begin{aligned}
P\left(\left\|\mathbf{X}_{n}\right\|_{D} \leqslant d\right)= & \frac{2}{d+2} \sum_{k=1, \text { odd }}^{d+1} \cos ^{n}\left(\theta_{d+2, k}\right) \frac{1+\cos \left(\theta_{d+2, k}\right)}{1-\cos \left(\theta_{d+2, k}\right)} \\
& -\frac{2}{d+1} \sum_{k=1, \text { odd }}^{d} \cos ^{n}\left(\theta_{d+1, k}\right) \frac{1+\cos \left(\theta_{d+1, k}\right)}{1-\cos \left(\theta_{d+1, k}\right)} .
\end{aligned}
$$

The trigonometric sums (28) and (29) are equivalent to the formulae presented in Proposition 14 and 15 of [14]. Compared to [14] the representations of (28) and (29) are more compact and show directly the symmetry with respect to $p$ and $q$. The computational complexity of (29) is four times less than that of the formula provided in Proposition 15 of $[14]$.

\section{Conclusion}

The main contributions of this paper are two novel approaches to tackle the combinatorial problem of counting the number of binary walks on $\mathbb{Z}$ of a certain range. This problem was pointed out by Feller [5] to be difficult. Both approaches rely on Hermann Weyl's discrepancy norm. The first exploits the geometric representation of its unit ball as a zonotope and reduces the original problem on $\mathbb{Z}$ to a known path-enumeration problem on a bounded lattice. The second approach exploits a Chebychev alternation property of this norm in order to prove the instructive Lost Walker Lemma, which allows an intuitive understanding of the problem. Compared to the martingale approach of [14], the outlined approaches surprise because of their simplicity and conciseness. The resulting formula for the distribution as a trigonometric sum is, after applying the eigenvalue decomposition of the adjacency matrix, more concise and less computationally complex than that of [14]. A combinatorial treatment of the moments and the mode of the resulting distribution is left for future research.

\section{References}

[1] P. Bauer, U. Bodenhofer, and E. P. Klement. A fuzzy algorithm for pixel classification based on the discrepancy norm. In Proc. of 5th IEEE Int. Conf. on Fuzzy Systems, volume III, pages 2007-2012, New Orleans, LA, 1996.

[2] B. Chazelle. The discrepancy method: randomness and complexity. Cambridge University Press, New York, NY, USA, 2000. 
[3] G. M. Cicuta, M. Contedini, and L. Molinari. Enumeration of simple random walks and tridiagonal matrices. Journal of Physics A: Mathematical and General, 35(5): 1125-1146, 2002.

[4] A. Dvorestzky and P. Erdös. Some problems on random walk in space. Second Berkeley Symp. Math. Statist. Prob., pages 353-368, 1951.

[5] W. Feller. The asymptotic distribution of the range of sums of independent random variables. Ann. Math. Statist., 22:427-432, 1951.

[6] S. Felsner and D. Heldt. Lattice path enumeration and Toeplitz matrices. In Proc. of 7th intern. conf. on lattice path combinatorics and applications (Lattice'10). July 4th-7th, Siena, Italia, 2010.

[7] N. C. Jain and S. Orey. On the range of random walk. Israel J. Math., 6:373-380, 1968.

[8] B. Moser. A similarity measure for image and volumetric data based on Hermann Weyl's discrepancy. IEEE Trans. Pattern Analysis and Machine Intelligence, 33(11):2321-2329, 2011.

[9] B. Moser. Geometric characterization of Weyl's discrepancy norm in terms of its ndimensional unit balls. Discrete and Computational Geometry, 48(4):793-806, 2012.

[10] B. Moser and T. Natschläger. On stability of distance measures for event sequences induced by level-crossing sampling. IEEE Trans. on Signal Processing, 2014, in press.

[11] H. Neunzert and B. Wetton. Pattern recognition using measure space metrics. Technical Report 28, University of Kaiserslautern, Department of Mathematics, November 1987.

[12] H. Niederreiter. Random number generation and quasi-Monte Carlo methods. Society for Industrial and Applied Mathematics, Philadelphia, PA, USA, 1992.

[13] F. Spitzer. Principles of random walks. Van Nostrand, New York, 1964.

[14] P. Vallois. The range of a simple random walk on $\mathbb{Z}$. Adv. Appl. Prob., 28:1014-1033, 1996.

[15] H. Weyl. Über die Gleichverteilung von Zahlen mod. Eins. Mathematische Annalen, 77:313-352, 1916.

[16] W. C. Yueh. Eigenvalues of several tridiagonal matrices. Appl. Math. E-Notes, 5:66$74,2005$. 